\title{
Dificultades y apoyos emocionales del alumnado migrante: un estudio de caso en un instituto multicultural
}

\author{
Esperanza-María Ceballos-Vacas \\ Universidad de La Laguna, España \\ mail: eceballodull.edu.es \\ ORCID: https://orcid.org/0000-0002-3714-1670
}

\author{
Elisa Trujillo-González \\ Universidad de La Laguna, España \\ mail: etrujillaull.edu.es \\ ORCID: https://orcid.org/0000-0002-4875-1087
}

\section{RESUMEN}

Se presenta un estudio de caso de un centro de ESO caracterizado por su elevada multiculturalidad. Para explorar las dificultades y los apoyos emocionales del alumnado migrante se realizaron entrevistas a la comunidad educativa y se analizaron documentos clave (PEC y PGA). Las principales dificultades percibidas fueron la experiencia migratoria, los conflictos ante el rol de género de la mujer occidental y la pertenencia a grupos segregados; seguidos por el desconocimiento del idioma, la falta de implicación en la relación escuela-familia y la desatención de las familias hacia sus hijas e hijos y, en menor medida, la discriminación social. Así mismo, se encontraron como apoyos la educación intercultural y emocional del centro, la acogida escolar al alumnado migrante y, en menor medida, la supervisión y apoyo escolar de la familia, así como la promoción de la relación escuela-familia. Se destaca la relevancia de la cultura escolar de cuidados al alumnado y sus familias, señalando las necesidades de formación del profesorado en competencias interculturales y emocionales y de relación con las familias migrantes.

Palabras Clave: adolescencia, educación emocional, educación intercultural, migrantes, necesidades.

\section{Emotional difficulties and support of migrant students: a case study in a multicultural secondary school}

\section{ABSTRACT}

In this paper, a case study is presented on a Compulsory Secondary Education school, characterized by high multiculturalism. Interviews to agents of the educative community were carried out to explore the emotional difficulties experienced by migrant students, and the support they received. In addition, key documents (School Educational Project and Annual General Program) were analyzed. The main difficulties perceived for migrant students were the migratory experience, conflicts regarding the gender role of Western women, and belonging to segregated groups. These were followed by lack of knowledge of the language, lack of involvement in the school-family relationship, and neglect of families towards their children, as well as social discrimination, to a lesser extent. Likewise, intercultural and emotional education and the school's reception of migrant students were found as emotional supports. Furthermore, the school's supervision and family support, together with the promotion of a school-family relationship, were also identified as sources of support for students, although to a lesser extent. The school culture of caring for students and their families, as well as the teachers' needs to be trained in intercultural and emotional skills in relation to migrant families are especially highlighted.

Keywords: adolescence, emotional education, intercultural education, migrants, needs.

ISSN: 0210-2773

DOI: https://doi.org/10.17811/rifie.50.4.2021.767-776 


\section{Introducción}

La globalización ha provocado importantes movimientos migratorios, convirtiéndose España en un país receptor desde los años 90 y Marruecos en el país de origen más recurrente (INJUVE, 2020). En el curso 2018-2019 se alcanzó un 9,9\% de alumnado extranjero, cuyas dificultades académicas no han pasado inadvertidas, promoviéndose iniciativas de apoyo idiomático, adaptación del currículum, etc. (Arroyo-González y Berzosa-Ramos, 2018). Sin embargo, se ha prestado menor atención a sus necesidades emocionales durante su aculturación: el proceso de aprendizaje de las claves culturales del recién estrenado entorno físico y social y la inmersión en un nuevo centro educativo ante un sistema escolar diferente, otro idioma, otros horarios y normas sociales... (Patel et al., 2016).

Con este estudio de caso se pretende ilustrar cómo ha vivido este desafío un centro de secundaria multicultural, ofreciendo el retrato experiencial de un instituto ante las necesidades emocionales de su alumnado migrante.

\section{Las dificultades emocionales del alumnado migrante}

Ser migrante y adolescente constituye en sí mismo un factor de riesgo, pues implica hacer frente a múltiples adaptaciones: las propias de la adolescencia y las relacionadas con la experiencia migratoria (Patel et al., 2016). La separación del contexto geográfico, familiar y social de origen y la aceptación de la nueva cultura implican un inmenso reto estructural y emocional (Sánchez-Castelló et al., 2020). Por otro lado, uno de los logros adolescentes más importantes es establecer buenas relaciones con iguales (Pegalajar-Palomino y Colmenero-Ruiz, 2014). No obstante, la pertenencia a un grupo socialmente desfavorecido puede complicar este objetivo en los centros escolares segregados étnica y económicamente (Murillo-Torrecilla et al., 2017).

Aunque la integración social sea lo deseable, las relaciones con personas de la misma cultura son positivas para ayudar a navegar entre nuevas normas y expectativas escolares, procurando identificación y "arropamiento" (Smith et al., 2020). También son clave para derribar las barreras idiomáticas que condicionan el éxito escolar y la autoestima (Arroyo-González y Berzosa-Ramos, 2018). Ante estos obstáculos no es de extrañar que el alumnado migrante disfrute de un círculo social más estrecho que el autóctono y que muestre menos habilidades emocionales y sociales (Pegalajar-Palomino y Colmenero-Ruiz, 2014).

La integración puede encontrar otro freno en las actitudes discriminatorias por razones culturales, raciales o de género, detectadas por Martin y Suárez-Orozco (2018) en cerca del 30\% de las aulas, si bien las agresiones más frecuentes consistieron en infravalorar la inteligencia y competencia del alumnado migrante. Estos prejuicios se producen asimismo entre los distintos grupos culturales migrantes, estableciéndose jerarquías que sitúan en el escalón más bajo al alumnado del norte de África y subsahariano (Mayoral-Peñas et al., 2020). Ante esta hostilidad, el blindaje en grupos de la misma cultura puede verse como un refugio seguro.

Sin embargo, también se pueden producir disonancias con la propia cultura. Es el caso de las jóvenes marroquíes que afrontan un conflicto entre su aspiración de mayor igualdad como mujeres del mundo occidental y su respeto a la cultura del honor (reputación social propia y del grupo cultural) (López-Zafra y El-Ghoudani, 2014). El triángulo migración-género-expectativas socioculturales y académicas sumerge a las adolescentes musulmanas en un dilema (Fernández-Larragueta et al., 2017), resuelto por aquellas con mayor inteligencia emocional a favor de la integración de ambas culturas (etnocultural y española) y no de la asimilación a la nueva (rechazando la propia), pues podrían dañarse sus lazos familiares y sociales (López-Zafra y El-Ghoudani, 2014).

Por otro lado, Lorenzo-Moledo et al. (2017) advierten sobre los prejuicios respecto a las familias migrantes que actúan de forma distinta, aunque no necesariamente negativa (p.e. en su vestimenta). No se debe obviar, no obstante, la situación de vulnerabilidad de estas familias, envueltas en un duro desafío de aculturación y dificultades (problemas económicos y laborales, falta de redes sociales, separación familiar...). Pues estas circunstancias críticas pueden alterar la necesaria priorización del cuidado y control familiar y rebajar las expectativas educativas sobre su descendencia (Passiatore et al., 2019; Patel et al., 2016).

Así mismo, pueden redundar en la menor implicación educativa de las familias extranjeras (Hernández-Prados et al., 2016), si bien no ha de deducirse irrevocablemente desafecto por la educación, pues existen múltiples barreras que paralizan su participación: La pobreza, el idioma, la incompatibilidad de horarios... aunque también se agregan los sentimientos de inferioridad y respeto ante el profesorado (Lorenzo-Moledo et al., 2017) y el choque cultural respecto al modelo de escuela experimentado en su país de origen. De ahí que los centros educativos deban asumir la iniciativa para que las familias migrantes se sientan bienvenidas, apeladas e implicadas (Hernández-Prados et al., 2016).

\section{Los apoyos emocionales al alumnado migrante}

Para mitigar estas dificultades y favorecer la adaptación del alumnado migrante, las administraciones educativas han establecido medidas compensatorias, sobre todo encaminadas a la inmersión lingüística. No obstante, el trabajo compartido entre la inmersión y el apoyo entre iguales en el aula ordinaria resulta más efectivo tanto para el aprendizaje lingüístico como para dar respuesta a las necesidades socio-emocionales (Arroyo-González y Berzosa-Ramos, 2018). Esto es importante, pues la falta de comunicación y de apoyo emocional-social podrían impulsar a asimilarse al nuevo contexto o a rechazarlo (Fernández-Larragueta et al., 2017; Viñuela-Hernández, 2019) en lugar de optar por la integración bicultural (López-Zafra y El-Ghoudani, 2014; Sánchez-Castelló et al., 2020).

Por esta razón, muchos centros han decidido incluir en el currículum la celebración de la diversidad cultural (Jaffe-Walter, 2018), facilitando que todo el alumnado reflexione sobre su propia cultura y se distancie de posiciones etnocéntricas (Fernández-Larragueta et al., 2017). Para ello es imprescindible que el profesorado mejore su competencia multicultural, mostrando tolerancia y sensibilidad acerca de las desigualdades interseccionales (económicas, educativas, de género...) de las familias migrantes (Martin y Suárez-Orozco, 2018). Pues para lograr una cultura escolar plenamente intercultural, se deben combatir los prejuicios y las actitudes discriminatorias (Passiatore et al., 2019), con un enfoque colaborativo y holístico en el que los equipos directivos son clave (Jaffe-Walter, 2018).

Esta ética de cuidados hacia el alumnado migrante y sus familias solo es viable cuando el profesorado se enraíza en la comunidad y entiende mejor los retos de las personas migrantes, tendiendo a una actitud más acogedora y comprensiva (Dabach et al., 2018). Además, cuando el alumnado migrante percibe que el profesorado le trata con justicia, le escucha y se preocupa por su bienestar, mejora su implicación y rendimiento, convirtiéndose así el centro escolar en un inestimable factor de resiliencia (Martin y Suárez-Orozco, 2018).

Por otro lado, la adolescencia es un buen momento para reforzar el aprendizaje de habilidades sociales (Domínguez-Alonso et 
al., 2015), aunque escasean las investigaciones sobre las ventajas de la educación emocional, especialmente en centros multiculturales (Mera-Lemp et al., 2014). En general, se enfatizan las carencias del profesorado, no sólo para educar emocionalmente, sino también para gestionar sus propias emociones (Bisquerra-Alzina y García-Navarro, 2018). Por tanto, resulta imprescindible potenciar el bienestar del profesorado y mejorar sus relaciones interpersonales en la escuela (Torrijos-Fincias y Martín-Izard, 2018) con programas de educación emocional centrados en el profesorado, el alumnado y su entorno (Pérez-Escoda et al., 2018).

Así mismo, deben revisarse las posibilidades reales de participación de las familias, pues muchos centros no incentivan su implicación (Lorenzo-Moledo et al., 2017). A ello se añaden las dificultades específicas de las familias migrantes y su situación de extrema vulnerabilidad económica y social. La participación familiar es causa y consecuencia del sentimiento de pertenencia a la comunidad escolar y solo es posible cuando las familias se encuentran con una comunidad "acogedora", que será tanto más decisiva cuanto más hostil y complicado sea el contexto (Hernández-Prados et al., 2019).

Por ello, Hernández-Prados et al. (2016) apuestan por la formación del profesorado en un modelo de participación escolar flexible y sensible, que atienda a la diversidad de condiciones socio-geográficas y familiares. Con más formación se favorece más la implicación y se comprenden mejor los distintos modelos culturales de crianza y socialización. Si el centro estimula la participación, las familias se involucran más, independientemente de su bagaje cultural, mostrando más interés por comunicarse con sus hijos e hijas y por supervisar sus tareas y felicitarles por sus logros, lo que resulta crucial para su adaptación (Gomariz-Vicente et al., 2019; Smith et al., 2020).

\section{Objetivo de investigación}

Este estudio de caso pretende conocer las dificultades emocionales del alumnado migrante y los apoyos recibidos a través de la percepción de su comunidad educativa y del análisis de la documentación del centro durante los cursos 2017/18 y 2018/19.

\section{El estudio de caso}

El centro se eligió por constituir un caso crítico (Flick, 2009), al reunir las características idóneas para un estudio relacionado con la multiculturalidad escolar.

\section{El escenario}

El Instituto se encuentra en un municipio del sur de Tenerife cercano a importantes enclaves turísticos de la isla. De acuerdo con el Instituto Canario de Estadística, el municipio contaba con un $46,8 \%$ y un $47,8 \%$ de población extranjera en 2018 y 2019. Los dos barrios más próximos registran un porcentaje aún mayor (alrededor del 52\%) (ISTAC, 2021).

El centro se inauguró apresuradamente en el curso 2002/03 con instalaciones prefabricadas provisionales (que aún permanecen) para hacer frente a las elevadísimas demandas de matrícula escolar en la zona. En el centro se imparte exclusivamente la ESO, acogiendo un promedio de 490 alumnas y alumnos (29,7 y 30.3\% migrantes de más de 30 nacionalidades) en los cursos 2017/18 y 2018/19. El origen marroquí es particularmente frecuente, así como el europeo (italiano, ruso...) y, en menor medida, el latinoamericano (colombiano, ecuatoriano...).

El claustro estuvo integrado por 44 docentes en el curso 2017/18 y por 46 en el 2018/19. El equipo directivo es estable desde el curso 2008/09. El instituto cuenta con una trayectoria relevante de respuesta a la multiculturalidad. Desde el primer curso creó su propio Programa de Educación Intercultural (PEI) y Plan de Apoyo Idiomático (PAI), desde el 2009/10 el Proyecto Somos de Colores (PSC) para mejorar la convivencia y desde el 2016/17 el Proyecto de Tutorías afectivas para alumnado en riesgo de abandono. El centro recibió el premio al mejor Proyecto de Educación Intercultural del Gobierno de Canarias en el curso 2007/08.

La participación de las familias es escasa. El AMPA sólo está constituida por tres madres. El nivel socioeconómico de la mayor parte de las familias es medio-bajo, ya que ocupan puestos de baja cualificación en la hostelería y el comercio con salarios bajos.

\section{Informantes clave}

- Del profesorado: Cuatro informantes hombres entre 26 y 35 (P1), entre 36 y 45 (P2 y P3) y entre 46 y 55 años (P5) y cuatro mujeres entre 36 y 45 (P6) y entre 46 y 55 años (P4, P7 y P8). P1 y P2 forman parte del equipo directivo y $\mathrm{P7}$ es la responsable del PSC.

- De la comunidad educativa externas al centro: La informante $\mathrm{C} 1$ es la trabajadora social del centro cultural juvenil, también es canaria y tiene entre 26 y 35 años. La C2 es la educadora social del distrito escolar, es canaria y tiene entre 36 y 45 años.

- Del alumnado: Tres informantes son migrantes, tienen 16 años y cursan $3^{\circ}$ de la ESO; son dos alumnas marroquíes (A1 y A2) y un alumno ruso (A3). El A4 es un alumno canario de 15 años que cursa $3^{\circ}$ de la ESO.

- De las familias: La informante M1 proviene de Siria, tiene entre 36 y 45 años y tres hijas y dos hijos. Una hija cursa $2^{\circ}$ de la ESO y un hijo $4^{\circ}$. La M2 es canaria y presidenta de la AMPA, tiene entre 46 y 55 años y una hija en $3^{\circ}$ de la ESO.

\section{Técnicas e instrumentos}

En este estudio solo se incluyen las preguntas para explorar las dificultades del alumnado migrante y los apoyos recibidos (ver Tabla 1), realizadas en el transcurso de entrevistas semiestructuradas más amplias sobre interculturalidad. Se seleccionaron así quince preguntas para el profesorado, dos para la trabajadora social y la educadora social, once para el alumnado migrante y tres para el canario, cinco para la madre migrante y tres para la presidenta de la AMPA. Por último, se analizaron como documentos clave el Proyecto Educativo de Centro (PEC) y la Programación General Anual (PGA).

\section{Procedimiento}

El equipo directivo del centro facilitó la colaboración del profesorado y alumnado (con permiso de sus familias) y los documentos clave. A la trabajadora social y a la educadora social se les pidió directamente su colaboración, así como a la presidenta de la AMPA. El contacto con la madre migrante se consiguió a través de la trabajadora social. Las entrevistas originales tuvieron una duración entre 30 y 60 minutos y se realizaron en español, garantizando su confidencialidad y anonimato.

\section{Análisis efectuados}

El análisis de las transcripciones de las preguntas de las entrevistas, así como del PEC y la PGA, se realizó reduciendo los datos textuales a unidades de análisis: categorías (y, en su caso, subcategorías) con el software Atlas.ti (versión 7.5.10). Para elaborarlas se siguió un proceso deductivo partiendo de la bi- 
Tabla 1.

Preguntas de las entrevistas semiestructuradas según el colectivo.

\begin{tabular}{|c|c|}
\hline Colectivo & Preguntas de las entrevistas semiestructuradas \\
\hline Profesorado & $\begin{array}{l}\text { ¿Con qué retos te has encontrado a la hora de dar clase a alumnado que proviene de otros sistemas educativos? } \\
\text { ¿Qué medidas de integración en el grupo-clase se llevan a cabo con el alumnado migrante? } \\
\text { ¿Cómo se ayuda al alumnado no hispanohablante en su aprendizaje del español? } \\
\text { ¿En qué grado crees que se siente integrado en el centro el alumnado migrante? } \\
\text { ¿Crees que, en general, si alguna alumna/o migrante tiene algún problema, puede contar con tu ayuda o con } \\
\quad \text { la de otro profesor o profesora del centro?, ¿cómo? } \\
\text { ¿Cómo se trabaja en el centro la educación emocional? } \\
\text { ¿Cómo se trabaja en el centro la educación intercultural? } \\
\text { ¿Qué actividades hace el centro para la integración del alumnado migrante? } \\
\text { ¿Cómo pueden apoyar la educación de sus hijos e hijas las madres y los padres migrantes? } \\
\text { ¿En qué grado crees que realizan dichas acciones las familias migrantes? } \\
\text { ¿Cómo podría apoyarse a las familias desde el centro? } \\
\text { ¿Cómo crees que se lleva la gente que vive en el barrio? } \\
\text { ¿Crees que hay grupos culturales cerrados al resto?, ¿cuáles? } \\
\text { ¿Las familias de la zona traen a sus hijas e hijos a este instituto?, ¿por qué? } \\
\text { ¿Cómo puede influir que en el centro haya tantas nacionalidades? }\end{array}$ \\
\hline Trabajadora social & $\begin{array}{l}\text { ¿Cómo es la convivencia en el barrio? } \\
\text { ¿Qué dificultades tienen los chicos y chicas del barrio? }\end{array}$ \\
\hline Educadora social & $\begin{array}{l}\text { ¿Qué barreras has detectado para la integración del alumnado migrante? } \\
\text { En cuanto al rol de la mujer, ¿has observado diferencias culturales en el instituto?, ¿cuáles? }\end{array}$ \\
\hline Alumnado migrante & $\begin{array}{l}\text { ¿Querías venir a vivir aquí? ¿Has venido con tu familia? } \\
\text { ¿Cómo te sentiste al llegar? ¿Cómo es ahora tu vida aquí? } \\
\text { ¿Qué es lo más difícil de irte a vivir a otro país? } \\
\text { ¿Qué te parece tu barrio? } \\
\text { ¿Te sientes canaria/o?, ¿por qué? } \\
\text { ¿Crees que debes valorar y conservar tu cultura?, ¿por qué? } \\
\text { ¿Crees que debes interesarte por la cultura de Canarias?, ¿por qué? } \\
\text { ¿Qué te parece tu escuela de aquí? } \\
\text { Cuando no entiendes algo en la escuela, ¿qué haces? } \\
\text { Si tienes algún problema personal, ¿qué haces? } \\
\text { ¿Crees que tus compañeras/os te comprenden a ti y a tu cultura?, ¿por qué? }\end{array}$ \\
\hline Alumnado canario & $\begin{array}{l}\text { ¿Qué te parece tu barrio? } \\
\text { ¿Qué es lo que más te gusta de vivir aquí? } \\
\text { ¿Y lo que menos? }\end{array}$ \\
\hline $\begin{array}{l}\text { Madre } \\
\text { migrante }\end{array}$ & $\begin{array}{l}\text { ¿Qué te parece tu barrio? } \\
\text { ¿Qué es lo que más te gusta de vivir aquí? } \\
\text { ¿Y lo que menos? } \\
\text { ¿Hasta qué punto crees que estás al tanto de lo que le pasa a tu hijo/a? } \\
\text { Cuando tu hija/o tiene algún problema en la escuela, ¿quién suele ir a hablar con el profesorado? }\end{array}$ \\
\hline $\begin{array}{c}\text { Presidenta de la } \\
\text { AMPA }\end{array}$ & $\begin{array}{l}\text { ¿Las familias de la zona traen a sus hijas e hijos a este instituto?, ¿por qué? } \\
\text { ¿Cómo puede influir que en el centro haya tantas nacionalidades? } \\
\text { ¿Qué actividades hace el centro para ayudar al alumnado migrante a integrarse? }\end{array}$ \\
\hline
\end{tabular}

Nota. Elaboración propia

bliografía anterior, completándolas y refinándolas de forma inductiva a través del análisis de los textos. A continuación, se relacionan las categorías y subcategorías junto a su código y su definición, organizadas en dos dimensiones de acuerdo con el objetivo del estudio: dificultades emocionales del alumnado y apoyos recibidos.

Dificultades emocionales:

- Experiencias migratorias (DE1): Dificultades aparejadas a la llegada al país de acogida, con las siguientes subcategorías: Aculturación (DE1.1), proceso de adaptación a la cultura de un nuevo país; choque cultural (DE1.2), diferencias entre la cultura del país de origen y de acogida; desarraigo (DE1.3), pérdida del contexto físico y social de origen; y separación familiar (DE1.4), división del núcleo familiar si sólo ha migrado el padre o la madre con toda o parte de su descendencia.

- Desconocimiento del idioma (DE2): Problemas para la comunicación, para las relaciones sociales y para el desempeño académico por no conocer el idioma.

- Discriminación (DE3): Rechazo o infravaloración sufridos por pertenecer a otra cultura.

- Grupos segregados (DE4): Presión para permanecer en grupos del mismo origen cultural con escaso nivel de apertura.

- Conflictos culturales ante el rol de género de la mujer occidental (DE5): Sentimientos contradictorios de las alumnas migrantes entre su deseo de adoptar roles occidentales como mujeres y la presión de su entorno cultural. 
- Desatención de las familias hacia sus hijas e hijos (DE6): Escasa inversión de tiempo y esfuerzo de las familias migrantes respecto al desarrollo y supervisión de sus hijos e hijas.

- Falta de implicación en la relación escuela-familia (DE7): Escasa colaboración y comunicación con el centro escolar.

Apoyos emocionales:

- Acogida escolar al alumnado migrante (AE1): Acciones del centro dirigidas a facilitar la integración escolar del alumnado migrante.

- Educación intercultural (AE2): Acciones educativas para la valoración de la diferencia, la convivencia y la igualdad.

- Educación emocional (AE3): Recursos que se emplean en el centro para promover el desarrollo emocional del alumnado, en especial, del alumnado migrante.
- Promoción de la relación escuela-familia desde el centro (AE4): Acciones del centro para apoyar a las familias migrantes y favorecer la relación escuela-familia.

- Supervisión y apoyo escolar de la familia (AE5): Implicación familiar en el desarrollo y educación de sus hijas e hijos.

\section{Resultados y discusión}

En las entrevistas y los documentos claves se han identificado 137 citas de dificultades emocionales (ver Figura 1) y 80 citas de apoyos emocionales (ver Figura 2) que se detallan, a continuación.

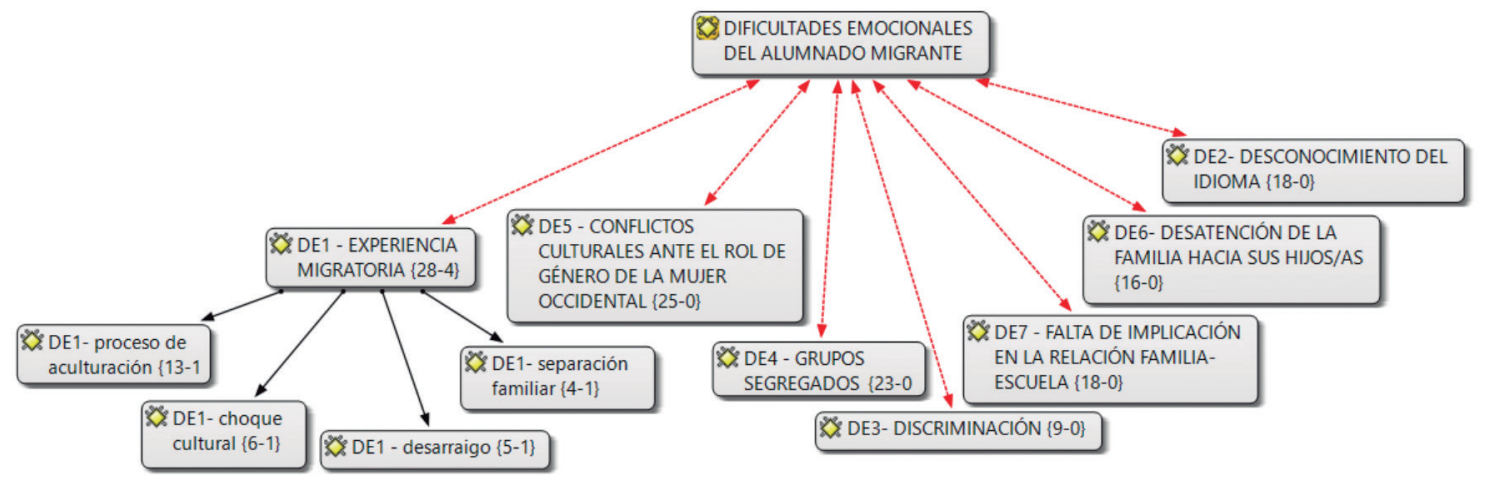

Figura 1. Categorías y subcategorías de dificultades emocionales del alumnado migrante. Nota. Elaboración propia con Atlas.ti

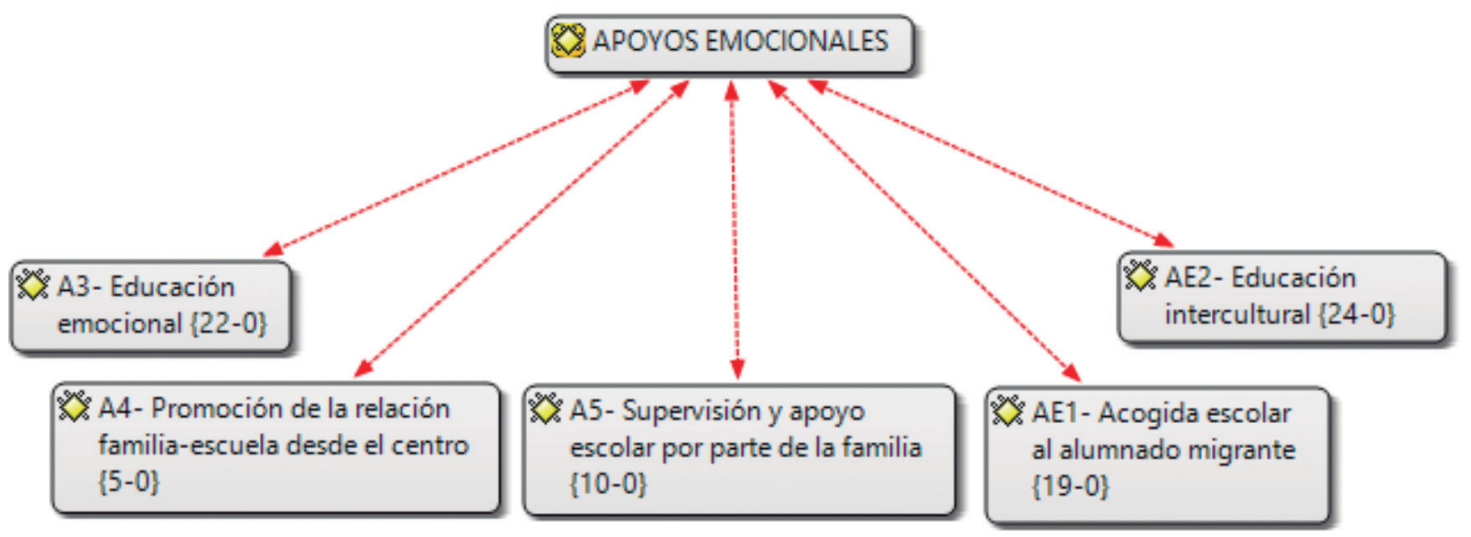

Figura 2. Categorías de apoyos emocionales al alumnado migrante. Nota. Elaboración propia con Atlas.ti

\section{Dificultades emocionales del alumnado migrante}

Las experiencias migratorias (28 citas) han acaparado la mayor parte de las alusiones, confirmándose como dificultades altamente estresantes (Sánchez-Castelló et al., 2020), concretadas en cuatro subcategorías. El proceso de aculturación (13 citas) poniendo énfasis en su complejidad (Patel et al., 2016): “el mayor reto es adaptarse a un nuevo país, a un nuevo idioma, a nuevas costumbres, etc." (P4-DE1). Lo que también se relaciona con la subcategoría desarraigo (5 citas), subrayando la incertidumbre y el duelo por lo dejado atrás: “...es como si empezaras todo de cero otra vez. Tampoco sabía aquí cómo sería la gente, si aceptarían nueva gente o no. La verdad, me resulta un poquito difícil. Dejar amigos. Dejar el instituto y la casa donde has estado toda tu vida. Tus recuerdos, tu cumpleaños y todo..." (A1-DE1.3).

Este desarraigo con frecuencia se magnifica por las situaciones incluidas en la subcategoría separación familiar (4 citas): "Hay numerosos casos en los que el núcleo familiar está dividido, encontrándose uno de los dos progenitores en el país de origen" (PEC 2018/2019-DE1.4); “aquí solamente mi padre, mi hermano 
y mi tío" (A2-DE1.4). Esta división familiar complica aún más la adaptación al nuevo contexto (Passiatore et al., 2019). Si bien el alumnado participante parece haber optado por un perfil bicultural, lo que puede favorecer su integración (López-Zafra y El-Ghoudani, 2014): “O sea, me siento parte de Marruecos y parte de aquí. Una mezcla" (A2-DE1.1). No obstante, la subcategoría choque cultural (6 citas) es reconocida por el alumnado: "me miran por llevar el pañuelo en la cabeza..." (A2-DE1.2) y por el profesorado: “En casa, los padres tienen unas costumbres, una visión del mundo. Y aquí, sus compañeros, la sociedad en la que ellos están viviendo, tienen otros estándares diferentes y hay un choque que tienen que aprender a llevar" (P6-DE1.2).

Esta colisión se hace particularmente patente respecto a los conflictos culturales ante el rol de género (25 citas). Las alumnas marroquíes del centro tienen que afrontar un dilema entre su cultura y la occidental (Fernández-Larragueta et al., 2017). Las reglas de la cultura del honor las sintetiza la madre siria: "No está bien, porque si una niña de catorce años se queda embarazada es un problema. Por eso no es bien [sic] que salgan solas cuando son pequeñas..." (M1-DE5). La presión social se ilustra en el fragmento de una de las alumnas marroquíes, mostrando cierta integración de ambas culturas, al juzgar ella también a otras compatriotas (López-Zafra y El-Ghoudani, 2014):

"Es ir por la calle y estar mirando a los lados por si hay algún moro. Es muy agobiante en verdad, pero lo peor es que ellos empiezan a criticar a las hijas de los demás, pero en verdad ellos no han visto a sus hijas. Sus hijas son peor [sic]. Sus hijas saben que ahí están sus padres, entonces por ahí no van. Así van por otro sitio y es peor cosa" (A2-DE5).

En este sentido, el instituto es descrito como un oasis donde se ha logrado una unión entre culturas que no existe más allá de los muros del centro: "Al final, esto es una burbuja en la que sí se está con alumnos de otros países, pero luego en su casa sigue siendo un mundo marroquí, colombiano o lo que sea" (P2-DE4). Estos grupos segregados (23 citas) del mismo origen cultural se encuentran claramente definidos en el barrio, afectando negativamente a la convivencia (Murillo-Torrecilla et al., 2017): “... siempre hay conflictos y todo eso. Siempre está la policía por ahí" (A4-DE4). La madre siria, al no pertenecer al grupo migrante mayoritario, abunda en esta cuestión: "Aquí hay muchos de Marruecos. $\mathrm{Y}$ no todos son buenos. $\mathrm{Y}$ a veces cuando ando con mi marido y mis hijos me miran, porque todos saben que soy de Siria. No soy de Marruecos. Y no soy de España" (M1-DE4).

Aunque se trata de la categoría menos frecuente, también se han detectado actitudes de discriminación (9 citas) entre el alumnado (Martin y Suárez-Orozco, 2018), que relegan al alumnado subsahariano y norteafricano al escalafón más bajo (Mayoral-Peñas et al., 2020): “Ha habido algunas cosas puntuales como negro o moro mierda... [sic]" (P7-DE3). Y también se producen, por parte de algunas familias, comentarios peyorativos en relación con la multiculturalidad que el centro combate en su línea de trabajo intercultural (Passiatore et al., 2019): “Más de una vez he tenido que pedirles a familias amablemente que no utilicen ciertas etiquetas para ciertos grupos sociales. Y te dicen: en la clase de mi hija hay muchas personas de aquí o de allá" (P1-DE3). La presidenta de la AMPA muestra su rechazo hacia estas actitudes xenófobas (Murillo-Torrecilla et al., 2017): “Sí. Hay muchos padres que los envían a otros institutos. Yo no lo entiendo. Tenemos un centro buenísimo. Unos profesores buenísimos. Yo siempre hablo bien del centro. No puedes despreciar a esta gente. Son personas igual que nosotros..." (M2-DE3).
A ello se une la dificultad inicial del alumnado migrante para entablar nuevas relaciones, por lo que su círculo social al principio es muy reducido (Pegalajar-Palomino y Colmenero-Ruiz, 2014), lo que provoca que busque refugio afectivo en su misma cultura (Smith et al., 2020):

"Cuando vine aquí los grupitos de amigos ya estaban hechos. Yo al principio me junté con una morita y ya luego me fui juntando más con los moros. Y con los españoles, en verdad, me quería juntar... Como que a lo mejor te acercas y dices: hola, qué tal, y te presentas y ya está, y ellos siguen más por su lado..." (A1-DE3).

El desconocimiento del idioma (18 citas) sobresale como una barrera para la adaptación social y el éxito académico (Arroyo-González y Berzosa-Ramos, 2018): "Primero no tenía amigos, no sabía el idioma” (A3-DE2); “...la verdad es que les cuesta muchísimo, al final hay que asumir que el proceso de adaptación les va llevar un año como mínimo" (P1-DE2). Esta limitación, junto a las carencias educativas, los problemas culturales y económicos y las diferencias con el modelo de escuela que conocen, también condiciona claramente la falta de implicación en la relación escuela-familia (18 citas) de las familias migrantes (Hernández-Prados et al., 2016):

"Hay muchas personas que no hablan español, entonces no se enteran de las circulares. Yo creo que no sienten el contexto porque su realidad es otra. Hemos hecho cosas en otros idiomas, pero al final también es algo cultural" (C2-DE7).

En el centro predomina el reconocimiento de los factores estructurales que constriñen a estas familias para apoyar el proceso educativo de sus hijas e hijos (Dabach et al., 2018): “Ellos quieren y les gustaría, pero no saben cómo" (P4-DE7); "muchas veces no tienen tiempo o no conocen el idioma o no tienen el nivel académico" (P6-DE7). Si bien también se pone en duda la prioridad de la educación para algunas familias y se señalan sus bajas expectativas sobre sus hijos e hijas (Patel et al., 2016): “Las expectativas de futuro hacia sus hijos no son lo positivas que deberían ser, creen que no pueden llegar a ser profesionales y piensan más a corto plazo y no invierten su tiempo en ayudarlos" (P8-DE7); "mis padres no saben que lo que importa es que termine los estudios" (A1-DE7)".

En esta misma línea, se sugiere cierta desatención de las familias hacia sus hijas e hijos (16 citas). No obstante, se alude a las dificultades de las familias (Passiatore et. al., 2019): “Los padres llegan aquí para intentar buscar un futuro y si tienen suerte lo consiguen. Si tienen problemas para conseguirlo, los niños terminan sin tener atención familiar adecuada" (P2-DE6). Las penosas condiciones de algunas familias son narradas por la madre siria:

"Mi marido hasta ahora no ha encontrado trabajo porque no tiene papeles. Y tengo muchos hijos. Por ahora estoy cogiendo una ayuda que se va acabar en octubre de 426 euros. Sólo tenemos una habitación y un salón. Estoy pagando para el alquiler 351 y me quedan sólo setenta y cinco para vivir todo el mes" (M1-DE6).

Otras familias disponen de trabajo, pero les faltan redes sociales para apoyarles en el cuidado de sus hijas e hijos:

"Un elevado número de familias de estos alumnos trabajan en hostelería, con salarios medios-bajos y horarios muy dispares, frecuentemente incompatibles con los horarios de 
los alumnos. En bastantes casos los alumnos se encuentran solos en momentos del día en que sería deseable que no fuese así" (PEC 2018/19-DE6).

\section{Apoyos emocionales que recibe el alumnado migrante}

Las acciones para responder a la diversidad cultural por medio de la educación intercultural han sido las más enunciadas (24 citas), dando cuenta de prácticas de una cultura escolar intercultural:

“Los profesores cuando llegan y ven la línea de trabajo que se lleva en este instituto, si no es su forma, intentan cambiarse. Hay de todo, pero se ha llegado a un grupo bastante numeroso de profesores bastante implicados y con sensibilización por este tema" (C1-AE2).

La integración del alumnado con un perfil bicultural es una de las máximas que guían la actividad diaria (López-Zafra y El-Ghoudani, 2014; Sánchez-Castelló et al., 2020): "la integración de otras culturas en la nuestra y también que no pierdan su cultura. Es fundamental" (P8-AE2). Para ello, el centro intenta involucrar a todo el alumnado y a sus familias en actividades en las que se valoran las culturas del alumnado migrante, contribuyendo a que todo el alumnado se distancie de posiciones etnocéntricas (Fernández-Larragueta et al., 2017): “Que, si no fuera por esas actividades, en verdad, cada uno estaría por su lado. Cada uno está con su grupito y ya está" (A1-AE2); "en las semanas culturales del centro trajimos mi madre y yo un par de platos de Marruecos y alguna vestimenta" (A2-AE2); "la bandera de Rusia está en el instituto" (A3-AE2). Esta visión de la multiculturalidad como factor de enriquecimiento cultural se combina con la prevención de conductas discriminatorias (Passiatore et al., 2019): “El profesorado está evitando ese tipo de comportamientos continuamente, pues intentamos que la integración del alumnado a nuestro sistema sea auténtica" (P4-AE2).

Estos ideales se intentan hacer extensivos a toda la comunidad escolar (Jaffe-Walter, 2018): “Es un paso imprescindible para la integración educativa, afectiva y social. Por ello se dispone de proyectos con el fin de sensibilizar al alumnado y a la comunidad educativa sobre la riqueza de la multiculturalidad" (P3-AE2). De hecho, el alumno canario apunta que lo que más le gusta de su barrio es que sea "multicultural... que hay gente de muchos sitios" (A4-AE2).

Estas acciones interculturales están claramente vinculadas a la acogida escolar al alumnado migrante (19 citas), iniciadas para dar respuesta al alumnado migrante recién llegado (Passiatore et al., 2019): "Fue la necesidad imperiosa de atender a ese alumnado lo que creó un Plan de Acogida y una especie de plan de integración en el centro ante un sistema educativo que no conocían absolutamente nada" (P5-AE1). Estas iniciativas se fueron desarrollando, teniendo en cuenta la inmersión lingüística y el apoyo entre iguales para favorecer su adaptación idiomática y social, lo que enlaza con lo apuntado por Arroyo-González y Berzosa-Ramos (2018), de forma que el alumnado aprenda el idioma de forma natural y además pueda sentir el respaldo del centro y del grupo-aula:

"Si habla otro idioma, intento buscar a alguien que le ayude. Le ubico en un grupo en que pueda ser bien acogido, en el que haya gente que pueda entender un poquito su cultura. Le pongo alumnos nexo. Un profesor que le sirva de nexo también" (P1-AE1).
La educación emocional (22 citas) es otra de las categorías más referenciada: "Fortalecer sus capacidades afectivas" (PEC 2017/18-AE3), sobre todo con el alumnado con riesgo de abandono escolar, para quien se puso en marcha el proyecto de "tutorías afectivas":

"El proyecto se apoya en la figura del tutor o tutora afectiva (con profesorado voluntario) y hace hincapié en la relación que se establece con el alumno o alumna, como una fuente de estabilidad emocional, que facilita su integración escolar y social" (PGA 2017/18-AE3).

La preocupación por el bienestar del alumnado, en general, es subrayada por el profesorado, de manera que pueda acudir al profesorado ante cualquier problema, lo que puede incidir positivamente en su implicación y rendimiento (Martin y Suárez-Orozco, 2018):

“Sí, sí. Es raro que no haya tocado alguno a la puerta: ¡Mira, me pasó esto!... es un mensaje que yo siempre intento lanzar para que siempre sepan que van a tener ahí a alguien que pueda ocuparse del problema, que un adulto puede resolver mejor que un niño" (P1-AE3).

Además, el profesorado parece ser consciente de la necesidad de trabajar las habilidades emocionales del alumnado, especialmente en contextos multiculturales (Mera-Lemp et al., 2014):

"Yo con mis alumnos siempre les hablo de empatía. Había un chico que era de China... era buenísimo, pero hablaba muy mal. Entonces, yo les decía a los chicos: imagínense que nos vamos a China y te ponen en un colegio que hablan solamente chino, que nadie habla español..." (P5-AE3).

No obstante, es preciso puntualizar que no se ha encontrado constancia de que el profesorado del centro disponga de formación en educación emocional, confirmando las carencias formativas apuntadas por Bisquerra-Alzina y García-Navarro (2018). Lo que recalca la importancia de mejorar sus competencias interculturales para mostrar sensibilidad y tolerancia tanto hacia las dificultades del alumnado migrante como hacia las de sus familias (Dabach et al., 2018). En este sentido, resulta fundamental comprender las desigualdades interseccionales de las familias migrantes (Martin y Suárez-Orozco, 2018). Por ello, es crucial que el centro amplifique las relaciones de cuidado hacia las familias, procurando la promoción de la relación escuela-familia desde el centro (5 citas): "Yo veo que el profesorado se vuelca. Llaman a las familias. Están muy pendientes y fomentan que las familias se impliquen" (C2-AE4). Esta categoría, no obstante, ha sido la menos aludida, si bien existe un proyecto de participación familiar centrado en la formación para que las familias apoyen educativamente desde sus hogares: "Queríamos enganchar a las familias para mejorar la convivencia con sus hijos en casa. Trabajamos el tema del acoso escolar, de la sexualidad en adolescentes, del consumo de estupefacientes..." (P4-AE4).

Aunque este proyecto está dirigido a todas las familias, desde el instituto se ha prestado atención específica a las familias migrantes: "Hicimos unos folletos informativos del centro, de lo que se estudiaba aquí y de cómo era el sistema educativo español. Fue la necesidad lo que creó un Plan de Acogida familiar" (P5-AE2). Así como cuidando que se sientan reconocidas, valoradas y apeladas para que sean capaces de implicarse (Gomariz-Vicente et al., 2019): 
“Por ejemplo, el día del migrante hemos traído algunas familias para que hablen de su experiencia y lo vean como algo cercano. Luego, cuando son días así de señalados, como Halloween, han venido familias que viven en diferentes sitios y ver cómo lo celebran. También las estamos invitando a que vengan a ver a sus hijos exponer en clase" (P7-AE4).

Por su parte, el alumnado migrante manifiesta que sus familias les intentan apoyar, a pesar de sus dificultades, lo que es decisivo para su adaptación (Gomariz-Vicente et al., 2019; Smith et al., 2020), quedando recogido en la categoría supervisión y apoyo escolar por parte de la familia (10 citas). Por ejemplo, el alumno ruso refleja la implicación de su familia: "Con mi padre si yo hago algo mal. Si a lo mejor suspender [sic] un examen, no se cabrea, pero me dice: que es para ti, para tu futuro" (A3-AE5). Asimismo, la madre siria intenta conocer las vivencias de su hija e hijo adolescentes: "Sí, sí, mi hijo me lo cuenta todo. Mi hija a veces no. Pero yo le veo en la cara que hay algo y ¿qué ha pasado? le grito a ella", animarles en sus estudios: "yo a mi hija le digo que estudie cuanto más mejor, que se dediquen en el futuro a lo que quieran, pero que sea una cosa buena" y mantener contacto con el centro: "voy yo al instituto si hay algún problema" (M1-AE5).

\section{Conclusiones}

La globalización ha creado entornos multiculturales que suponen un gran enriquecimiento cultural, siempre y cuando gocen de respeto y tolerancia hacia la diversidad (Passiatore et al., 2019). Las instituciones educativas están obligadas a promover una convivencia cultural armoniosa, reconociendo las numerosas dificultades que rodean al alumnado migrante y a sus familias (Sánchez-Castelló et al., 2020). Además, la ESO se desarrolla en plena adolescencia (Domínguez-Alonso et al., 2015), por lo que al alumnado migrante se le acumulan los retos que debe afrontar (Patel et al., 2016), jugando un papel fundamental la comunidad educativa para su superación.

En general, los resultados del estudio indican que el centro tiende a una cultura escolar que celebra la diversidad cultural, comprende las dificultades interseccionales de las personas migrantes y combate la discriminación (Passiatore et al., 2019). Estas premisas interculturales, que huyen de las habituales prácticas etnocéntricas, asimilacionistas e invisibilizadoras de otras culturas (Arroyo- González y Berzosa-Ramos, 2018; Fernández-Larragueta et al., 2017) llevan una trayectoria estable en el instituto y ponen de relieve el papel decisivo de los equipos directivos (Jaffe-Walter, 2018). Sin embargo, aunque atenuadas, siguen presentes más dificultades (más citas) que apoyos emocionales, sobre todo en lo relacionado con la experiencia migratoria (Patel et al., 2016). Especial relevancia adquieren los conflictos con el rol de género en las alumnas marroquíes, que hallan su mejor equilibrio en la integración bicultural (López-Zafra y El-Ghoudani, 2014).

Aunque el centro está preocupado por el bienestar del alumnado migrante y sus familias, e incluso existen algunos programas centrados en lo emocional (Tutorías Afectivas para alumnado en riesgo de abandono escolar), no queda constancia de un trabajo globalizado al respecto ni de una formación explícita del profesorado en competencias interculturales ni emocionales, lo que coincide con lo observado por Bisquerra-Alzina y García-Navarro (2018) y Martin y Suárez-Orozco (2018). Esto es importante porque, para que la escuela pueda actuar como un auténtico factor de resiliencia del alumnado migrante y de sus familias, es preciso que la comunidad educativa (sobre todo el profesorado) esté, además de sensibilizada, formada en dichas competencias.
Otra asignatura pendiente es la mejora de la relación escuela-familia, en especial con las familias migrantes. Si bien se manifiesta cierta consciencia de las dificultades interseccionales de estas familias para su participación (Dabach et al., 2018), acompañada de buenas intenciones e iniciativas (p.e. Plan de Acogida Familiar), se constata la necesidad de una formación y una intervención más sistemáticas para lograr una participación escolar flexible y sensible a la diversidad (Hernández-Prados et al., 2016).

Para finalizar, este estudio presenta las limitaciones propias de un estudio de caso. Se trata de una mirada local, aunque profunda, en cuanto a la comprensión de las dificultades y los apoyos emocionales del alumnado migrante. Sin embargo, evidencia que un instituto con recursos limitados, pero con una cultura escolar comprometida con la educación intercultural, puede ser eficaz para responder al desafío de la multiculturalidad.

\section{Referencias bibliográficas}

Arroyo-González, Ma-José \& Berzosa-Ramos, Ignacio (2018). Atención educativa al alumnado inmigrante: En busca del consenso. Revista de Educación, 379, 192-203.

Bisquerra-Alzina, Rafael \& García-Navarro, Esther (2018). La educación emocional requiere formación del profesorado. Participación educativa, 5(8), 13-28.

Dabach, Dafney-Blanca; Suárez-Orozco, Carola; Hernández; Sera-J. \& Brooks, Maneka- Deannad (2018). Future Perfect?: Teachers' Expectations and Explanations of their Latino Immigrant Students' Postsecondary Futures. Journal of Latinos and Education, 17(1), 38-52.

Domínguez-Alonso, José, López-Castedo, Antonio, Álvarez-Roales, Enrique (2015). Implicación de variables sociales y educativas en la conducta asertiva adolescente. Aula abierta, 43(1), 26-31.

Fernández-Larragueta, Susana, Fernández-Sierra, Juan \& Rodorigo, Monia (2017). Expectativas socioeducativas de alumnas inmigrantes: Escuchando sus voces. Revista de Investigación Educativa, 35(2), 483-498.

Flick, Uwe (2009). An introduction to qualitative research. Sage

Gomariz-Vicente, Mํa-Ángeles, Martínez-Segura, Ma-José \& Parra-Martínez, Joaquín (2019). Desde la implicación en el hogar de las familias a la facilitación de los docentes en un contexto multicultural. Revista Electrónica Interuniversitaria de Formación del Profesorado, 22.

Hernández-Prados, Mํ-Ángeles; Gomariz-Vicente, $\mathrm{M}^{\mathrm{a}}$-Ángeles; Parra-Martínez, Joaquín y García-Sanz, Mª-Paz (2016). Familia, inmigración \& comunicación con el centro escolar: Un estudio comparativo. Educación XX1, 19(2), 127-151.

Hernández-Prados, Ma-Ángeles, García-Sanz, Ma-Paz, Parra-Martínez, Joaquín \& Gomariz-Vicente, Má-Ángeles (2019). Participación familiar en los centros de educación secundaria. Anales de Psicología, 35(1), 84-94.

INJUVE (2020). Informe Juventud en España 2020. Dirección General del INJUVE y Observatorio de la Juventud en España.

ISTAC (2021). Población según sexos y países de nacimiento. Municipios por islas de Canarias y años. ISTAC: Estadísticas de la Comunidad Autónoma de Canarias

Jaffe-Walter, Reva (2018). Leading in the Context of Immigration: Cultivating Collective Responsibility for Recently Arrived Immigrant Students. Theory Into Practice, 57(2), 147-153.

López-Zafra, Esther \& El-Ghoudani, Karima (2014). The influence of culture of honor and emotional intelligence in the acculturation of Moroccan immigrant women. The Spanish Journal of Psychology, 17, 1-9. 
Lorenzo-Moledo, Mar, Godás-Otero, Agustín \& Santos-Rego, Miguel-A. (2017). Main determinants of immigrant families' involvement and participation in school life. Cultura y Educación, 29(2), 213-253.

Martin, Margary \& Suárez-Orozco, Carola (2018). What It Takes: Promising Practices for Immigrant Origin Adolescent Newcomers. Theory Into Practice, 57(2), 82-90.

Mayoral-Peñas, Milagros, Jiménez-Blasco, Beatriz-Cristina, Sassano-Luiz, Silvana \& Resino-García, Rosa (2020). Inmigración y educación: experiencias de discriminación en la Comunidad de Madrid. Espacio Tiempo y Forma. Serie VI, Geografía, 13, 191-214.

Mera-Lemp, Ma-José, Martínez-Taboada, Cristina, \& Elgorriaga-Astondoa, Edurne (2014). Rendimiento académico, ajuste escolar e inteligencia emocional en adolescentes inmigrantes y autóctonos. Boletín de psicología, 110, 69-82. https://www. uv.es/seoane/boletin/previos/N110.htm

Murillo-Torrecilla, F-Javier, Martínez-Garrido, Cynthia y Belavi, Guillermina (2017). Segregación escolar por origen nacional en España. OBETS. Revista de Ciencias Sociales, 12(2), 395-423.

Patel, Sita, Clarke, Anette, Eltareb, Fazia, Macciomei, Erynn, \& Wickham, Robert (2016). Newcomer Immigrant Adolescents: A Mixed-Methods Examination of Family Stressors and School Outcomes. School Psychology Quarterly, 31(2), 163-180.

Passiatore, Ylenia, Pirchio, Sabine, Carrus, Giuseppe, Maricchiolo, Fridanna, Fiorilli, Fiorilli, Caterina \& Arcidiacono, Francesco (2019). Intercultural practices and inclusive education in Europe: Can migration be a resource for individual and societal development? European Journal of Psychology of Education, 34(1), 209-224.

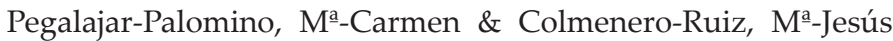
(2014). Inteligencia emocional en alumnado de Educación Secundaria en contextos multiculturales. Electronic Journal of Research in Educational Psychology, 12(2), 325-342.

Pérez-Escoda, Nuria, Filella-Guiu, Gemma, Alegre-Roselló, Albert, \& Bisquerra-Alzina, Rafael (2018). Desarrollo de la competencia emocional de maestros y alumnos en contextos escolares. Electronic Journal of Research in Education Psychology, 10(28), 1183-1208.

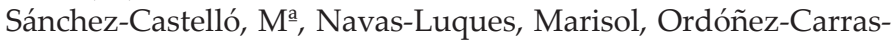
co, Jorge, \& Rojas-Tejada, Antonio-J. (2020). Acculturation and adaptation of adolescents with immigrant backgrounds in Spain: Psychosocial profiles of latent classes. Revista De Psicologia Social, 35(3), 560-588.

Smith, Naila, Brown, Joshua, Tran, Tran, \& Suárez-Orozco, Carola (2020). Parents, friends and inmigrant youths' academic engagement: A mediation analysis. International Journal of Psychology, 55(5), 743-753.

Suárez-Orozco, Carola; Casanova, Saskias; Martin, Margary; Katsiafica, Dalal; Cuellar, Veronica; Smith, Naila-Antonia \& Dias, Sandra-Isabel (2015). Toxic Rain in Class: Classroom Interpersonal Microaggressions. Educational Researcher, 44(3), 151-160.

Torrijos-Fincias, Patricia, Martín-Izard, Juan-Francisco, \& Rodríguez-Conde, $\mathrm{M}^{\mathrm{a}}$-José (2018). La educación emocional en la formación permanente del profesorado no universitario. Profesorado, Revista de Currículum y Formación del Profesorado, 22(1), 579-597.

Viñuela-Hernández, Ma-Paulina, Rodríguez-Menéndez, Carmen, Torío-López, Susana \& Fernández-García, Carmen (2019). Práctica discursiva familiar y cultura contra escolar en minorías étnicas. Aula Abierta, 48(3), 279-290. 
